\title{
Non-Reagent Cleaning of Industrial Wastewater, Containing Heavy Metals Based on Technology of Hydrothermodynamic Cavitation
}

\author{
Olga G. Dubrovskaya* and Vladimir A. Kulagin \\ Siberian Federal University \\ 79 Svobodny, Krasnoyarsk, 660041, Russia
}

Received 16.02.2019, received in revised form 12.04.2019, accepted 30.05.2019

The results of studies on the conditioning systems of industrial wastewater containing heavy metal ions are presented. An analysis of the physicochemical modifications of the component composition of the model runoff has been carried out and the effectiveness of reducing the content of heavy metal ions under various hydrothermodynamic modes has been determined. Methods for mathematical modeling of determining the modes of optimal cavitation treatment of runoff are proposed, the advantages of upgrading cleaning equipment with the inclusion of SC reactors are identified.

Keywords: industrial wastewater, heavy metals in stock, activation, cavitation, cavitation technology, recycled water use, wastewater treatment.

Citation: Dubrovskaya O.G., Kulagin V.A. Non-reagent cleaning of industrial wastewater, containing heavy metals based on technology of hydrothermodynamic cavitation, J. Sib. Fed. Univ. Eng. technol., 2019, 12(4), 460-467. DOI: 10.17516/1999494X-0153.

\section{Безреагентная очистка промышленных сточных вод, содержащих тяжелые металлы на основе технологии гидротермодинамической кавитации}

О.Г. Дубровская, В.А. Кулагин Сибирский федеральнылй университет Россия, 660041, Красноярск, пр. Свободный, 79

Изложены результаты исследований систем кондиционирования промышленных сточных вод, содержаших ионы тяжельх металлов. Проведен анализ физико-химических модификаций компонентного составамодельного стока и определена эффективность снижения содержания

(C) Siberian Federal University. All rights reserved

This work is licensed under a Creative Commons Attribution-NonCommercial 4.0 International License (CC BY-NC 4.0).

* Corresponding author E-mail address: dubrovskayaolga@mail.ru 
ионов тяжельх металлов при различных режимах гидротермодинамического воздействия. Предложены способы математического моделирования определения режимов оптимальной кавитационной обработки стока, выявлень преимущества модернизации очистного оборудования с включением узла СК-реакторов.

Ключевые слова: промышленные сточные воды, тяжелье металль в стоке, активация, кавитация, кавитационные технологии, оборотное водопользование, очистка сточных вод.

\section{Введение}

Основной задачей, направленной на снижение негативного экологического воздействия промышленных сточных вод на водные объекты, является разработка систем оборотного водопользования [1-5]. Однако возвратные воды часто не могут быть использованы для систем оборотного водопользования даже после очистки, так как эффективность стандартных методов кондиционирования промышленного стока недостаточно высока. Наибольшую актуальность представляет проблема очистки стоков, содержащих ионы тяжелых металлов [6-16]. Как правило, такие сточные воды характерны для промышленных предприятий приборостроения, машиностроения, металлургии. Тяжелые металлы представляют серьезную экотоксикологическую опасность, их негативное воздействие на физиологию человека было отмечено неоднократно.

На локальных очистных сооружениях вышеперечисленных предприятий чаще всего применяют реагентные методы очистки стока, которые не только не позволяют достичь эффективности очистки до норм сброса в природные водоемы рыбохозяйственного значения, но и остаточные концентрации велики для сброса в централизованную городскую канализацию. Сброс таких вод вызывает экономические потери, так как без тщательной очистки он запрещен и, как правило, приводит предприятия к наложению штрафов. Важно, что присутствие ионов тяжелых металлов может влиять на значения рН и электропроводности раствора, а химические соединения вызывают негативное воздействие на работу инженерных коммуникаций: подвергаясь гидролизу, они могут действовать как сильные кислоты, усиливать вспенивание стока, участвовать в формировании накипей [1]. В связи с этим целесообразно разрабатывать и внедрять в производство альтернативные, безреагентные, инновационные и высокоэффективные методы очистки сточных вод, содержащих ионы тяжелых металлов. К таким методам можно отнести очистку стока, основанную на эффектах гидротермодинамической кавитации [2-5].

Цель исследования - научное обоснование и разработка безреагентной технологии очистки сточной воды, содержащей ионы тяжелых металлов, с применением методов гидротермокавитационного воздействия, происходящего на границах раздела фаз в исследуемой среде, и подбор оптимальных режимов кавитационной обработки сточной воды с целью ее эффективной очистки.

\section{Методология проведения работы}

Методологической базой являются экспериментальные методы термодинамических, кинетических, технологических исследований. В качестве эмпирической базы исследования применены лабораторные и стендовые полупромышленные кавитационные установки. При исследовании эффективности очистки стока использовались стандартные методики оценки качества воды, методы термографического анализа состава образующихся осадков, методы математического моделирования и интерпретации результатов исследования.

$$
-461-
$$




\section{Результаты исследования и их обсуждение}

Изменение физико-механических свойств жидкости при гидротермодинамическом кавитационном воздействии основано на высокой концентрации (кумуляции) энергии в очень небольшом объеме газообразной и жидкой среды с последующим ее высвобождением в критически малом временном периоде.

Физические характеристики жидкости оказывают различное влияние на интенсивность кавитационного воздействия, увеличивая или уменьшая скорость кумулятивных струек вблизи твердых границ потока. Особенно сильно это влияние сказывается на последнем этапе схлопывания пузырьков, когда их размеры очень малы. Увеличение вязкости и плотности снижает интенсивность кавитационного воздействия; силы поверхностного натяжения ускоряют коллапс пузырьков; наличие растворенных и нерастворенных газов в жидкости замедляет этот процесс, демпфируя соударение стенок пузырька. Поэтому дегазация жидкости служит одним из способов интенсификации кавитационного воздействия.

Изменение условий проведения технологических процессов также может существенно влиять на интенсивность кавитационного воздействия, а следовательно, и на скорость протекания соответствующего процесса. Например, снижение температуры и давления насыщенных паров повышает интенсивность кавитационного воздействия. Повышение давления увеличивает скорость кумулятивной струйки при схлопывании пузырька, однако при значительном повышении статического давления трудно получить режимы развитой кавитации.

Время кавитационной обработки также неоднозначно влияет на конечный результат. Не всегда увеличение времени обработки ведет к увеличению технологического эффекта. Поэтому, применяя гидродинамическую кавитацию, необходимо учитывать не только физические свойства жидкостей, но и условия проведения конкретного технологического процесса: давление, температуру, длительность обработки, степень турбулентности потоков, наличие твердых частиц и т. д.

В качестве объекта исследования использовались модельные растворы, основные физикохимические показатели которых обоснованы реальными характеристиками промышленных соков. Исходные концентрации модельных растворов были заданы на основании количественного химического анализа (КХА) сточной воды цехов предприятия приборостроения. Исходные концентрации загрязнителей и требования к остаточным концентрациям очищенного стока с целью его повторного применения приведены в табл. 1.

Как упоминалось выше, в качестве альтернативного метода очистки воды от ионов тяжелых металлов предложен способ гидротермодинамической кавитации. При этом использовали две независимые линии исследований на суперкавитационных реакторах (СК-реактор) разного типа. Такая постановка исследовательской задачи изучения воздействия гидротермодинамической кавитации на сточную воду позволила получить наиболее адекватные результаты по воспроизводимости условий эксперимента, а также дала возможность проведения измерений физических параметров в кавитационной области. Заметим, что физико-химические эффекты идентичны независимо от способа возбуждения кавитации, что, безусловно, важно при решении вопроса о выборе СК-реакторов для определенных технологических решений.

Доказано [1-5], что в условиях гидротермодинамической кавитации протекают сложные физико-химические процессы, классифицируемые следующим образом:

$$
-462-
$$


Таблица 1. Требования к качеству воды для электронной промышленности РФ ОСТ 11.029.003-80 и США по ASTM D-5127-90

Table 1. Water quality requirements for the electronic industry of the Russian Federation OST 11.029.003-80 and the USA according to ASTM D-5127-90

\begin{tabular}{|c|c|c|c|c|c|c|c|c|}
\hline \multirow{3}{*}{ Показатель } & \multirow{3}{*}{$\begin{array}{c}\text { Исходная } \\
\text { концентрация } \\
\text { мг/дм }{ }^{3}\end{array}$} & \multicolumn{7}{|c|}{$\begin{array}{c}\text { Требования к оборотной технической воде } \\
\text { предприятий приборостроения }\end{array}$} \\
\hline & & \multicolumn{3}{|c|}{$\begin{array}{l}\text { ОСТ } 11.029 .003-80 \\
\text { КАТЕГОРИИ }\end{array}$} & \multicolumn{4}{|c|}{$\begin{array}{l}\text { ASTM D-5127-90 } \\
\text { КАТЕГОРИИ }\end{array}$} \\
\hline & & A & Б & B & E-1 & E-2 & E3 & E4 \\
\hline $\mathrm{pH}$ & 8,7 & \multicolumn{3}{|c|}{$6,9-7,2$} & \multicolumn{3}{|c|}{7,0} & 7,5 \\
\hline $\mathrm{Cu}$ & 3,94 & 0,002 & 0,002 & 0,003 & 0,001 & 0,001 & 0,002 & 0,5 \\
\hline $\mathrm{Zn}$ & 1,25 & - & - & - & 0,5 & 1 & 5 & 500 \\
\hline $\mathrm{Pb}$ & 0,22 & - & - & - & - & - & - & - \\
\hline $\mathrm{Fe}$ & 3,7 & 0,015 & 0,02 & 0,03 & - & - & - & - \\
\hline $\mathrm{Ni}$ & 2,52 & & & & 0,1 & 1 & 2 & 500 \\
\hline $\mathrm{Mn}$ & $\mathbf{0 , 0 3}$ & - & - & - & - & - & - & - \\
\hline $\mathrm{Cr}^{6+}$ & 0,012 & - & - & - & - & - & - & - \\
\hline
\end{tabular}

- окислительно-восстановительные реакции с участием присутствующих в водной среде органических и неорганических веществ за счет образования в растворе $\mathrm{H}_{2} \mathrm{O}_{2}, \mathrm{O}_{2}, \mathrm{O}_{3}, \mathrm{O}$ и $\mathrm{OH}^{-}$;

- цепные реакции в растворе, инициируемые продуктами расщепления присутствующих в растворе примесей;

- деструкция макромолекул и инициирование деполимеризации полимерных соединений;

- реакции между растворенными газами внутри кавитационных пузырьков.

Так, в качестве обоснования направления очистки от тяжелых металлов был произведен анализ образовавшегося промежуточного скоагулированного вещества и последующий анализ образовавшегося осадка.

Эффективность очистки при различных режимах кавитационной обработки представлена в табл. 2. Математическое моделирование процесса кавитационной обработки стока и подбор оптимального режима представлены совокупностью методик визуального программирования - VBA, сочетаемого с вычислительными возможностями Excel (рис. 1, рис. 2).

На основании матриц изменения концентрации ионов тяжелых металлов в очищаемой сточной воде и полученных зависимостей от таких факторов как скорость вращения кавитацонной крыльчатки, время обработки, рН среды, температуры среды получены уравнения регрессии второго порядка. По уравнениям регрессии получены графические интерпретации в виде поверхностей (рис. 2), что позволяет регулировать процесс безреагентного кондиционирования сточных вод, содержащих тяжелые металлы и учитывать в дальнейшем технико-экономические показатели. При обсчете данных использован алгоритм оценки коэффициентов нелинейной регрессионной модели методом наименьших квадратов. Представлен оценочный критерий y1-2 в факторном пространстве следующих 
Таблица 2. Эффективность удаления тяжелых металлов при различных режимах кавитации

Table 2. The removal efficiency of heavy metals in various modes of cavitation

\begin{tabular}{|c|c|c|c|c|c|c|c|c|c|c|}
\hline \multirow{2}{*}{ ион } & \multirow{2}{*}{$\begin{array}{c}\text { Исходная } \\
\text { концентрация } \\
\text { мг/дм }\end{array}$} & \multicolumn{5}{|c|}{ Концентрация ионов после кавитационной обработки } \\
\cline { 3 - 12 } & $\mathbf{3}$ & $30 \mathrm{c}$ & $60 \mathrm{c}$ & $90 \mathrm{c}$ & $30 \mathrm{c}$ & $60 \mathrm{c}$ & $90 \mathrm{c}$ & $30 \mathrm{c}$ & $60 \mathrm{c}$ & $90 \mathrm{c}$ \\
\hline $\mathrm{pH}$ & $\mathbf{8 . 7}$ & 8,2 & 8,2 & 7,9 & 7,8 & 7,2 & 8,1 & 8,8 & 8,8 & 8,8 \\
\hline $\mathrm{Cu}$ & $\mathbf{3 , 9 4}$ & 3,55 & 2,95 & 2,55 & 2,68 & 1,73 & 2,45 & 2,75 & 2,75 & 2,75 \\
\hline $\mathrm{Zn}$ & $\mathbf{1 , 2 5}$ & 0,87 & 0,87 & 0,87 & 0,8 & 0,8 & 0,9 & 0,83 & 0,83 & 0,83 \\
\hline $\mathrm{Pb}$ & $\mathbf{0 , 2 2}$ & 0,143 & 0,143 & 0,143 & 0,127 & 0,068 & 0,149 & 0,149 & 0,149 & 0,149 \\
\hline $\mathrm{Fe}$ & $\mathbf{3 , 7}$ & 2,66 & 2,40 & 2,06 & 1,92 & 0,4 & 0,6 & 2,66 & 2,66 & 2,66 \\
\hline $\mathrm{Ni}$ & $\mathbf{2 , 5 2}$ & 1,58 & 1,58 & 1,58 & 1,53 & 0,63 & 1,43 & 1,86 & 1,86 & 1,86 \\
\hline $\mathrm{Mn}$ & $\mathbf{0 , 0 3}$ & 0,022 & 0,019 & 0,017 & 0,0156 & 0,003 & 0,0048 & 0,022 & 0,022 & 0,022 \\
\hline $\mathrm{Cr}{ }^{6+}$ & $\mathbf{0 , 0 1 2}$ & 0,010 & 0,01 & 0,010 & 0,004 & 0,004 & - & 0,01 & 0,01 & 0,01 \\
\hline
\end{tabular}

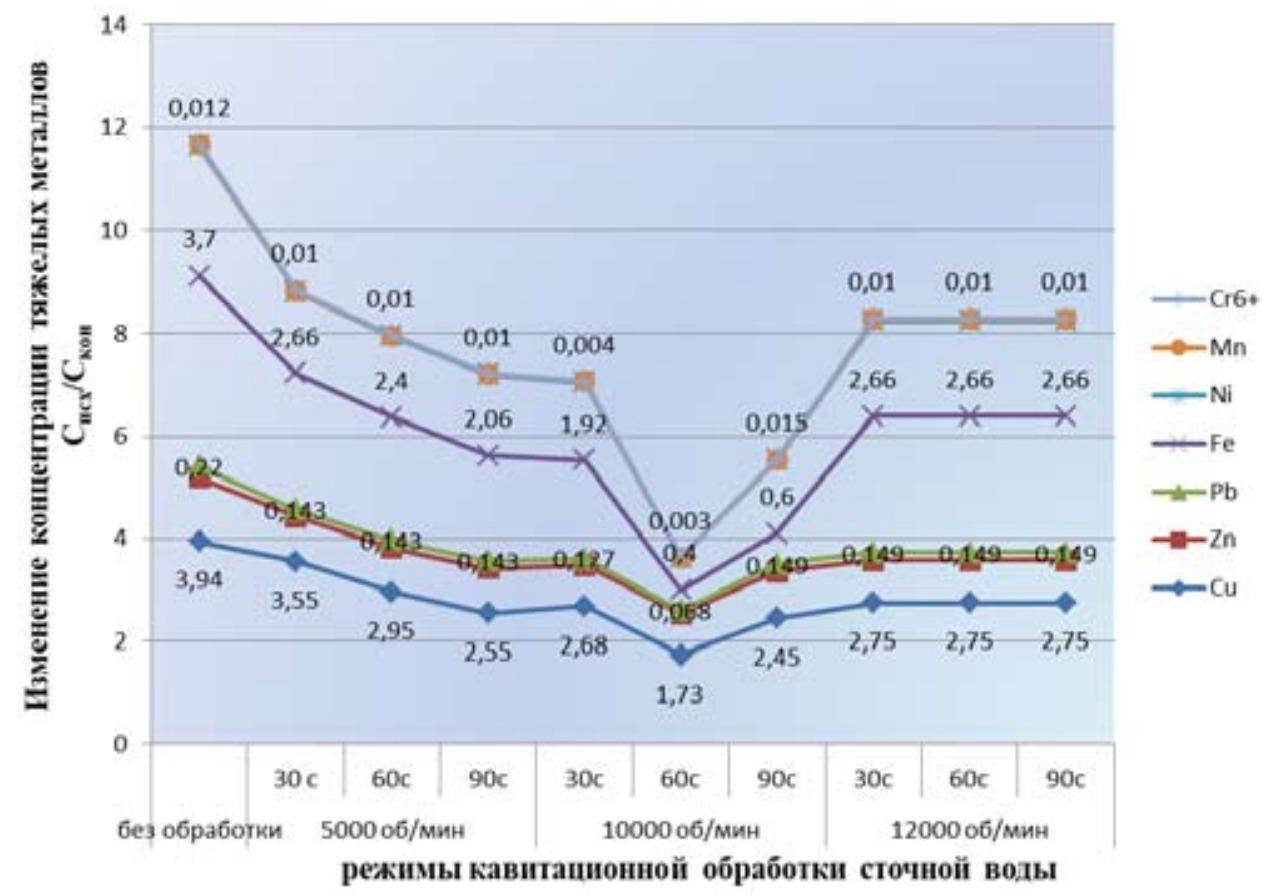

Рис. 1. Зависимость эффективности удаления ионов тяжелых металлов от режимов кавитационной обработки сточной воды

Fig. 1. The dependence of the efficiency of removal of heavy metal ions from the modes of cavitation treatment of waste water

параметров: $x 1 ; x 2 ; x 3$. На рис. 2 представлена регулировочная диаграмма очистки стока на примере иона меди.

Интерпретируя результаты математического моделирования и сопоставляя их с фактическими изменениями концентраций ионов тяжелых металлов при различных режимах гидротермодинамической обработки модели сточной воды, можно сделать вывод, что режимом 


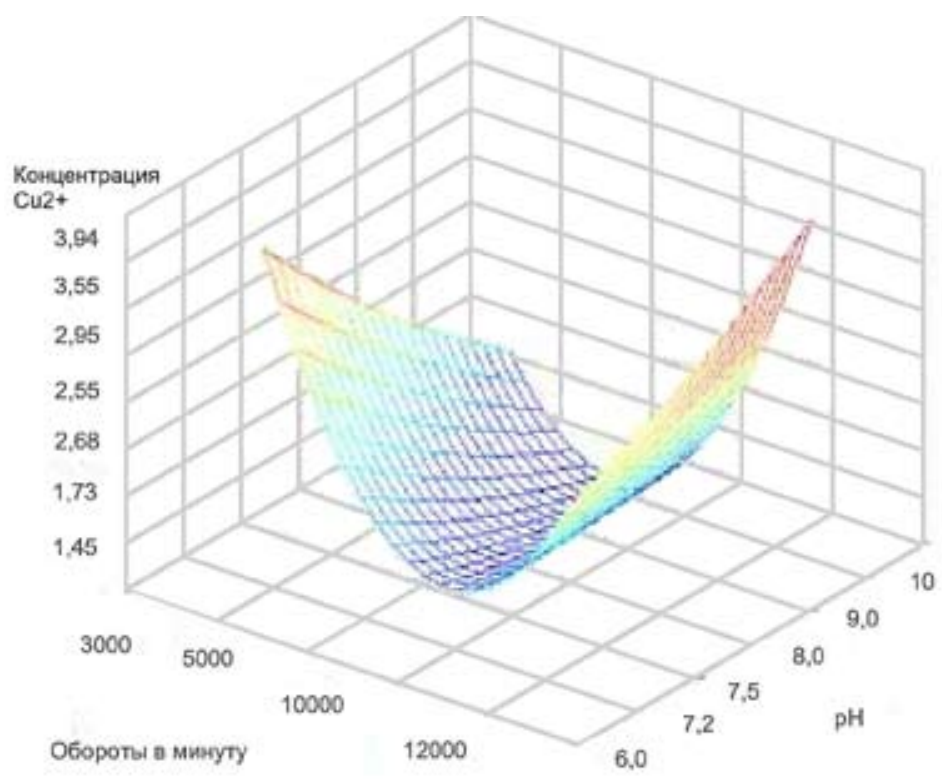

Рис. 2. Регулировочная диаграмма процесса очистки сточных вод, содержащих ионы тяжелых металлов (на примере иона $\mathrm{Cu}^{2+}$ ) методом кавитационной обработки

Fig. 2. Adjusting diagram of the process of wastewater treatment containing heavy metal ions (for example, $\mathrm{Cu}^{2}+$ ion) by cavitation treatment

оптимальности для удаления комплекса ионов тяжелых металлов является обработка при 10000 оборотов в минуту в течение 60 секунд. При более интенсивном режиме кавитационного воздействия наблюдается образование промежуточных продуктов окисления металлов и перекомбинация активных ионов в водной среде с образованием скоагулированых структур в виде гидроксидов металлов - например, $\mathrm{Fe}(\mathrm{OH})_{3}$ или же гидроксоаквакомплексов, таких как $\left[\mathrm{Cu}\left(\mathrm{OH}_{2}\right)_{2}(\mathrm{OH})_{2}\right]^{0},\left[\mathrm{Zn}\left(\mathrm{OH}_{2}\right)_{2}(\mathrm{OH})_{2}\right]^{0},\left[\mathrm{Ni}\left(\mathrm{OH}_{2}\right)_{2}(\mathrm{OH})_{2}\right]^{0},\left[\mathrm{Ni}\left(\mathrm{OH}_{2}\right)_{4}(\mathrm{OH})_{2}\right]^{0},\left[\mathrm{Fe}\left(\mathrm{OH}_{2}\right)(\mathrm{OH})_{3}\right]^{0}$, $\left[\mathrm{Fe}\left(\mathrm{OH}_{2}\right)_{3}(\mathrm{OH})_{3}\right]^{0}$. Данные вещества, в свою очередь, обладая слоем электростатического притяжения за счет изменения заряда комплексона - от положительного до отрицательного (при pH свыше 10.0), выступают коагулянтами. Образующийся скоагулированный осадок в рамках данного исследования также был проанализирован. Определены размеры частиц осадка турбидиметрическим и седиментационными методами анализа. Так, размер хлопьев осадка скоагулированных на гидроксоаквакомплексах $\mathrm{Ni}, \mathrm{Cu}, \mathrm{Zn}$ достигает 130-140 нм, тогда как хлопья осадка, образующиеся и седиментированные на комплексах иона $\mathrm{Fe}^{3+}$ составяют от 24 до 50 мкм.

Все исследования проведены в Исследовательской лаборатории Инженерно-строительного института СФУ (ИЛ СМиХАВ) при совместной работе с Китайско-Российской Международной лабораторией кавитационных технологий.

\section{Заключение}

Данные технические решения позволяют достичь требуемого качества воды для повторного использования. Главными достоинствами предлагаемого безреагентного метода на основе эффектов гидротермодинамической кавитации являются:

$$
-465-
$$


1) возможность очистки различного стока с различным качественно-количественным содержанием загрязняющих веществ;

2) возможность использования предлагаемой технологии при модернизации существующего оборудования. СК-генераторы легко встраиваются в существующие сооружения - например, аккумулирующие емкости, без изменения их конструкции;

3) возможность автоматизировать процесс очистки;

4) снижение эксплуатационных экономических затрат.

Введение блока кавитационной обработки для очистки промышленного стока и кондиционировании технической воды позволит сформировать замкнутый оборотный цикл водопользования и значительно снизить эксплуатационные затраты предприятия. Результаты данного исследования могут быть применены в локальных очистных установках предприятий с целью очистки сточной воды от ионов тяжелых металлов.

\section{Благодарность}

Исследование выполнено при финансовой поддержке РФФИ и Правительства Красноярского края в рамках научного проектов № 18-48-242001, 18-41-242004 и 18-41-242008.

\section{Список литературы}

[1] Дубровская О.Г., Приймак Л.В., Андруняк И.В. Ресурсосберегающие технологии обезвреживания и утилизации отходов предприятий теплоэнергетического комплекса Красноярского края: монография, Красноярск: Сиб. федер. ун-т, 2014. 164 с. [Dubrovskaya O.G., Priymak L.V., Andrunyak I.V. Resource-saving technologies for decontamination and waste disposal of enterprises of the heat and power complex of the Krasnoyarsk Territory., Krasnoyarsk: Sib. Fed. Univ., 2014. 164 (in Russian)].

[2] Дубровская О.Г., Евстигнеев В.В., Кулагин В.А. Кондиционирование сточных вод энергетических систем и комплексов. J. Sib. Fed. Univ. Eng. technol., 2011, 4(6), 665-675 [Dubrovskaya O.G., Evstigneev V.V., Kulagin V.A. Conditioning of wastewater energy systems and complexes. J. Sib. Fed. Univ. Eng. technol., 2011, 4(6), 665-675 (in Russian)].

[3] Дубровская О.Г., Кулагин В.А Интенсификация процессов сорбционной очистки нефтесодержащих сточных вод с использованием гидротермодинамических эффектов кавитации, Журнал СФУ. Техника и технологии, 2016, 9(2), 268-279 [Dubrovskaya O.G., Kulagin V.A. Intensification of the processes of sorption purification of oily wastewater using hydrothermodynamic effects of cavitation, J. Sib. Fed. Univ. Eng. Technol, 2016, 9(2), 268-279 (in Russian)].

[4] Дубровская О.Г., Кулагин В.А., Сапожникова Е.С. Современные компоновки технологических схем очистки сточных вод с использованием кавитационной технологии, Журнал СФУ. Техника и технологии, 2015, 8(2), 217-223 [Dubrovskaya O.G., Kulagin V.A., Sapozhnikova E.S. Modern layout of technological schemes of wastewater treatment using cavitation technology, J. Sib. Fed. Univ. Eng. Technol, 2015, 8(2), 217-223 (in Russian)].

[5] Дубровская О.Г., Кулагин В.А., Сапожникова Е.С. и др. Математическое моделирование кавитационных процессов при кондиционировании промышленных сточных вод, Журнал СФУ. Техника и технологии, 2015, 8(3), 369-376 [Dubrovskaya O.G., Kulagin V.A., Sapozhnikova E.S. 
Mathematical modeling of cavitation processes in the conditioning of industrial wastewater, J. Sib. Fed. Univ. Eng. Technol, 2015, 8(3), 369-376 (in Russian)].

[6] Гимаева А.Р., Валинурова Э.Р., Игдавлетова Д.К., Кудашева Ф.Х. Сорбция ионов тяжелых металлов из воды активированными углеродными адсорбентами, Сорбционные и хроматографические прочессы, 2011, 11(3), 350-356 [Gimaeva A.R., Valinurova E.R., Igdavletova D.K., Kudasheva F.Kh. Sorption of heavy metal ions from water by activated carbon adsorbents, Sorption and chromatographic processes, 2011, 11(3), 350-356 (in Russian)].

[7] Зыкова И.В., Лысенко И.В., Панов В.П. Адсорбция ионов меди керамической крошкой из бинарных и многокомпонентных растворов, Известия вузов. Химия и химическая технология, 2004, 47(9), 151-167 [Zykova I.V., Lysenko I.V., Panovv V.P. Adsorption of copper ions by ceramic chips from binary and multicomponent solutions, Proceedings of universities. Chemistry and Chemical Technology, 2004, 47(9), 151-167 (in Russian)].

[8] Цветкова А.Д., Акаев О.П. Исследование процесса адсорбции ионов меди на модифицированном диоксиде кремния, Вестник КГУ им. Н.А. Некрасова, 2011, 2, 27-30 [Tsvetkova A.D., Akaev O.P. Investigation of the process of adsorption of copper ions on modified silicon dioxide, Bulletin of KSU named after. N.A. Nekrasov, 2011, 2, 27-30 (in Russian)].

[9] Мухин В.М. Тарасов А.В., Клушин В.Н. Активные угли России. М.: Металлургия. 2000. 352 c. [Mukhin V.M., Tarasov A.V., Klushin V.N. Active coals of Russia. M.: Metallurgy. 2000. 352 p. (in Russian)].

[10] Тимофеев К.Л., Усольцев А.В., Краюхин С.А., Мальцев Г.И. Кинетика сорбции ионов индия, железа и цинка слабокислотными катионитами, Сорбционные и хроматографические проиессы, 2015, 15(5), 720-729 [Timofeev K.L., Usoltsev A.V., Krayukhin S.A., Mal'tsev G.I. Kinetics of sorption of indium, iron, and zinc ions by weakly acidic cation exchangers, Sorption and chromatographic processes, 2015, 15(5), 720-729 (in Russian)].

[11] Филатова Е.Г., Помазкина О.И., Дударев В.И., Шевелева Н.Н. Комплексная технология извлечения ионов никеля и еди из промышленных сточных вод, Водоочистка, 2012, 12, 20-25 [Filatova E.G., Pomazkina O.I., Dudarev V.I., Sheveleva N.N. Complex technology for the extraction of nickel ions and one from industrial wastewater, Water treatment, 2012, 12, $20-25$ (in Russian)].

[12] Wang Ji-Zhong, Sheng-Rong Li, Bao-Lin Liu, Jing-Gui Tong. Removal of heavy metals from wastewater using natural minerals, Bull Mineral Petrol Geochem, 2005, 24(2), 159-164.

[13] Svilovi'c S., Ruši'c D., Stipiši'c R. Modeling batch kinetics of copper ions sorption using synthetic zeolite NaX, Journal of Hazardous Materials, 2009, 170, 941-947.

[14] Demirbas Ayhan. Heavy metal adsorption onto agrobased waste materials, Journal of Hazardous Materials, 2008, 157(2-3), 220-229.

[15] Lokendra S. Thakur, Parmar Mukesh. Adsorption of heavy metal from synthetic waste water by tea waste adsorbent, International Journal of Chemical and Physical Sciences, 2013, 2(6), 6-19.

[16] Tumin Najua D., Luqman Chuah A., Zawani Z., Abdul Rashid S. Adsorption of copper from aqueous solution by Elais Guineensis kernel activated carbon, Journal of Engineering Science and Technology, 2008, 3(2), 180-189. 